\title{
Evaluation of Contactless Human-Machine Interface for Robotic Surgical Training
}

\author{
Fabien Despinoy ${ }^{1,2} \cdot$ Nabil Zemiti $^{2}$ - Germain Forestier ${ }^{3}$ - Alonso \\ Sánchez $^{2}$ • Pierre Jannin ${ }^{1}$ • Philippe Poignet ${ }^{2}$
}

\begin{abstract}
Purpose Teleoperated robotic systems are nowadays routinely used for specific interventions. Benefits of robotic training courses have already been acknowledged by the community since manipulation of such systems requires dedicated training. However, robotic surgical simulators remain expensive and require a dedicated humanmachine interface.

Methods We present a low-cost contactless optical sensor, the Leap Motion, as a novel control device to manipulate the RAVEN-II robot. We compare peg manipulations during a training task with a contact-based device, the electro-mechanical Sigma.7. We perform two complementary analyses to quantitatively assess the performance of each control method: a metric-based comparison and a novel unsupervised spatiotemporal trajectory clustering.

Results We show that contactless control does not offer as good manipulability as the contact-based. Where part of the metric-based evaluation presents the mechanical control better than the contactless one, the unsupervised spatiotemporal trajectory clustering from the surgical tool motions highlights specific signature inferred by the human-machine interfaces.
\end{abstract}

This work was supported in part by the French ANR within the Investissements d'Avenir Program (Labex CAMI, ANR11-LABX0004); by the Equipex ROBOTEX Program (ANR10-EQPX-44-01); and by the Région Languedoc-Roussillon.

Fabien Despinoy $(\bowtie)$

fabien.despinoy@univ-rennes1.fr

1 LTSI - INSERM, UMR 1099, Université de Rennes

1, Rennes, F-35000, France.

2 LIRMM - CNRS, UMR 5506, Université de Montpellier, Montpellier, F-34000, France.

3 MIPS (EA 2332), Université de Haute Alsace, Mulhouse, F-68100, France.
Conclusion Even if the current implementation of contactless control does not overtake manipulation with high-standard mechanical interface, we demonstrate that using the optical sensor complete control of the surgical instruments is feasible. The proposed method allows fine tracking of the trainee's hands in order to execute dexterous laparoscopic training gestures. This work is promising for development of future human-machine interfaces dedicated to robotic surgical training systems.

Keywords Contactless Teleoperation · Hand Tracking · Human-Machine Interface · Robotic Surgical Training · Unsupervised Trajectory Analysis.

\section{Introduction}

The $d a$ Vinci system from Intuitive Surgical Inc. [13] is the most commonly used telesurgical robot in the operating room (OR). With 3,919 installed systems at the end 2016, it allowed to perform 753,000 procedures all over the world in 2016 (74.7\% of them in the US) [15]. Robotic-assisted surgery is becoming standard for some procedures such as hysterectomy, prostatectomy and partial nephrectomy [24].

From a practical point of view, telesurgery is directly beneficial for the surgeon and makes possible ergonomic control of the surgical instruments. However, manipulating such device implies some constraints that can lead to technical (i.e., limit of technology, robot or surgical instruments malfunction) and medical complications (i.e., patient damaging, longer recovery period) which are sometimes underestimated $[2,4]$. Thus, a dedicated training is required to safely handle surgical instruments.

In this context, similarly to the Fundamentals of Laparoscopic Surgery (FLS) that defines standard prac- 

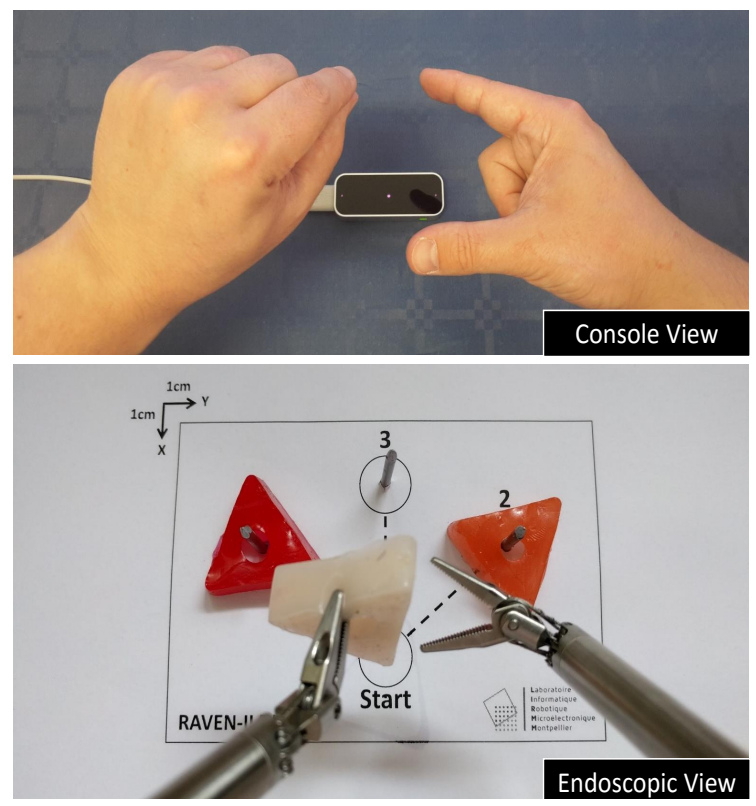

Fig. 1: The proposed contactless control approach with the Leap Motion interface and the RAVEN-II robot for surgical training.

tices for laparoscopic training and evaluation, a recent international program emerged to create standards for robotic-assisted training, education and assessment. The Fundamentals of Robotic Surgery (FRS) supports the large extension of robotic usage in the OR, leading to a real need for skill training [30], where surgical simulation platforms aim to prepare surgeons [28, 35]. Nevertheless, current training systems available in the market [25] are relatively expensive and difficult to use for residents and surgeons due to the limited access (i.e., availability and number of devices) in the different medical centers. To increase availability of training systems for continuous teaching, low-cost technologies are required. In this paper we propose to compare the performance of a new low-cost contactless interface with respect to a high-quality mechanical interface in order to control a telesurgical robot (fig. 1). In extension, these humanmachine interfaces (HMIs) can be employed to handle robotic surgical simulators.

\section{Related Work}

Gesture motions is the most accurate and efficient communication channel when the objective is to finely control instrument tooltips $[31,42]$. In this context, two different approaches can be identified. The first approach relies on the pose of the hand as raw input to control a robot while the second approach uses a specific hand pose dictionary to classify in real-time the hand pose linked to a robot action [20,27]. Taking into account the large amount of gesture possibilities and the required accuracy to safely control a surgical robot, this paper focuses on the first approach that uses the hand pose information as input to control surgical instruments. Addressing hand pose robot control, multiple works have been done with the Kinect For Windows from Microsoft. Du et al. [9] showed that the proposed contactless approach was suitable to control an industrial robot with the hand pose information for a basic translation task. Additionally, Dragan et al. [8] compared the use of a Kinect device with other mechanical HMIs to accurately control robot motions for a complex task, taking into account online replanification for semi-autonomous execution. However, those works highlight the limitations of the Kinect for hand motion control where both version were initially designed for a complete whole body tracking. Consequently, these sensors do not provide enough accuracy and repeatability for fine hand and finger tracking.

Dedicated to hand and finger tracking, recent devices showed promising results, especially the Leap Motion. Hernoux et al. [17] used the Leap Motion sensor to track one human finger and mimic the writing motions with an industrial robot. In the surgical field, Vargas et al. [36] used the Leap Motion to control simulated surgical tools with the index finger only. Zhou et al. [41] compared the use of the Kinect, the Leap Motion and other devices to control the Taurus robot (equipped with a scalpel). However, the control of the surgical tool was not complete since they extracted the position from the operator's hand to control the instrument position only. Travaglini et al. [34] employed the Leap Motion to control a 6 Degrees of Freedom (DoFs) concentric tube robot and evaluated the performance of this interface during a pituitary tumor resection task on phantoms. Control of the robot tooltip was achieved using the hand pose only. Their results showed that the Leap Motion is a confident HMI even if furthers developments are required to precisely handle surgical instruments.

The present work deals with a complete 7 DoFs contactless interaction to accurately control surgical instruments. Here, we focus on fine motions that are required for high-quality execution of dexterous surgical gestures. From Despinoy et al. [7], such approach was presented and a feasibility study with a qualitative assessment was achieved. We therefore propose a modified hand tracking model to improve mapping between the surgical tool and the operator's hand. Furthermore, we propose a quantitative evaluation of the surgical instrument motions when executing a pick-and-place training task using the RAVEN-II surgical robot, a dual-arm $d a$ 
Vinci-like system dedicated to research. This analysis is performed using two different quantitative approaches. Where the first approach relies on metric-based computations that provide raw scores of the performance, the second one is inspired by the works from Forestier et al. [10-12]. We develop a novel method to analyse spatiotemporal variations between trajectories and use an unsupervised clustering method to quantify similarity between surgical instrument motions. With such method, we want to analyse if similar manipulability is required for the design of HMIs dedicated to surgical training, in contrast to perioperative ones.

The remaining sections are organized as follows. In Section 3 , we present the robotic setup and the novel contactless control approach, as well as the methodological pipeline from the user hand tracking to the robot control. Section 4 presents both the dataset acquired during the surgical training task and the metrics. Additionally, we describe a complementary evaluation based on a novel unsupervised spatiotemporal trajectory clustering for a quantitative analysis of surgical tool motions. Section 5 details the results obtained for both analyses. Finally, we discuss our setup and results in Section 6 .

\section{Teleoperation Setup}

For an effective comparison of both HMIs, the RAVENII robotic platform from Applied Dexterity has been used. Similar to a dual-arm da Vinci system, this teleoperation system is composed of two parts: the console and the robot (fig. 2). In the following, we describe first both interfaces to control the robot (Section 3.1). Then we develop the method to accurately control surgical tools with the Leap motion (Section 3.2). The last section describes how the Raven-II robot is controlled to achieve the teleoperation task (Section 3.3).

\subsection{Human-Machine Interfaces}

The console (or master manipulator side) is visible at the left in fig. 2. It is composed of a controller, three screens, two Sigma.7 handles, an armrest and a foot pedal to manually activate and deactivate the remote robot (not present in the pictures). In the next, this foot pedal action will be referred as "clutch". The screens are used to display multiple information: at the center we show on a 3D compatible screen a high-quality endoscopic video feedback, whereas at the left and right sides we display information about the tracking and the robot status. For this experiment, we added one Leap Motion device on its support to compare manipulation with the Sigma.7 mechanical interface and the Leap
Motion contactless device.

On the one hand, two Sigma.7 devices from Force Dimension are attached to the structure and oriented towards the user in order to avoid any collision between handles during manipulation. This haptic device was designed to capture 7 DoFs from the hands including grasping. The hand configuration is presented at the left in fig. 3. Capable of reaching an accuracy of about $0.002 \mathrm{~mm}$ and a refresh rate at $4 \mathrm{kHz}$ through USB 2.0, this device fits the requirement for medical applications [33]. The main advantage of the Sigma.7 interface is the force feedback capability that can be used for haptic applications. For a fair comparison, this feature was disable during the experiment.

On the other hand, the Leap Motion device placed on a homemade support is a tracking system able to follow in real-time fingers and hands from the user. The device consists of two cameras and three infrared LED with a wavelength of 850 nanometers (outside the visible light spectrum). Relying on a closed API for body part tracking (Leap Motion SDK V2), the reported accuracy of the device is between $0.01 \mathrm{~mm}$ and $0.5 \mathrm{~mm}$ [14] with a decent refresh rate under $120 \mathrm{~Hz}$ using USB 2.0 interface. Originally dedicated to virtual reality manipulation, preliminary experiments have shown that this interface can reach the minimal requirements to be used for telesurgical manipulation $[7,41]$. For this device, the hand configuration is similar than the Sigma.7 as presented at the right in fig. 3 .

\subsection{Control with the Leap Motion}

The Sigma.7 has been designed for a complete surgical teleoperation experience and allows to fully control articulated instruments (i.e., pose and grasping angle). However, in the case of the Leap Motion, the mapping between hands and surgical tools is missing. While the main usage of this optical device is to point out or guide in virtual environments as a mouse can do, our objective is to find the best mapping strategy to fully control surgical instruments with intuitiveness and manipulability.

In this paper, we propose a $3 \mathrm{D}$ model to match hand pose with the surgical tool pose (fig. 4). For this purpose, the thumb, the index and the palm center of both hands have been used to create a robust model and mimic surgical grasper motions attached to the robot. Each finger represents one extremity of the tool where the pose of the palm center corresponds to the final articulation pose of the grasper. Where one could argue that the wrist center could better define the hand center of motion with respect to the instrument, preliminary experiments have shown that using this reference leads 

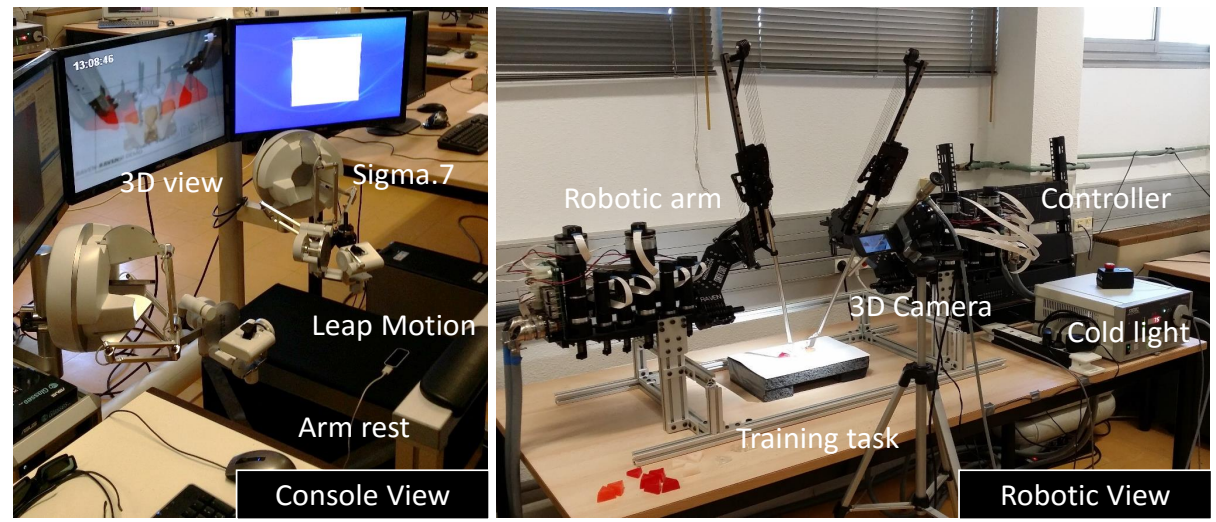

Fig. 2: The two views of the telesurgery setup. The left picture shows the entire console to handle the distant robot. The right picture presents the RAVEN-II telesurgical robot with all the equipments.
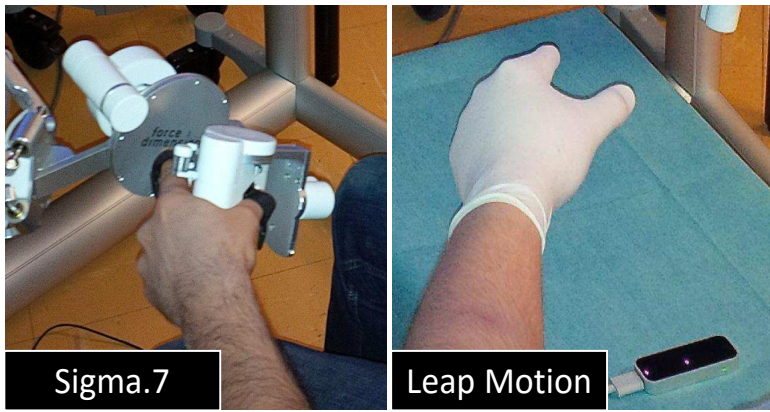

Fig. 3: Hand configuration with both the Sigma.7 and the Leap Motion devices.
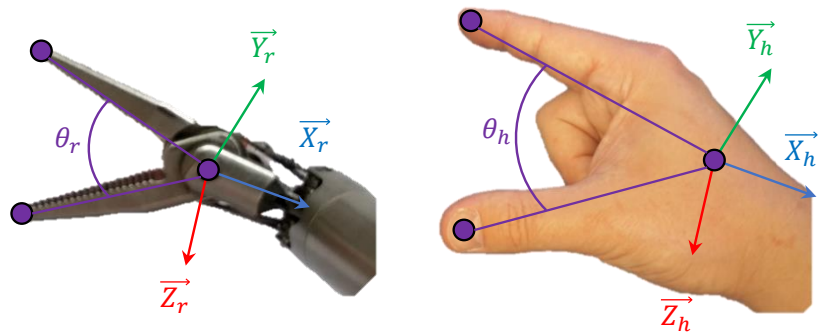

Fig. 4: Hand model mimics instrument.

to more disturbance when controlling the surgical tools (i.e. adding noise and unexpected translations at the end-effector). Finally, in order to be able to grab and release objects with the tools, the open and close motions have been mapped with the gap angle between fingers. Such configuration allows to fully interact with the environment and uses 7 DoFs, guaranteeing intuitiveness and precision during manipulation.

\subsection{Surgical Robot Control}

The robot (or slave side) is visible at the right in fig. 2 . The RAVEN-II robot is an open-source platform dedicated to research in robotic telesurgery [16]. Composed of two robotic arms, actuation of the surgical tools is achieved by 8 motors combined with a cable-driven mechanical architecture for a lighter structure. Each surgical tool allows 7 DoFs at the tooltip. The control software runs on top of the Robotic Operating System (ROS) middleware, on a real-time Linux kernel (Ubuntu 10.04 LTS with RT-Preempt patch).

Data computed from the console are directly filtered using a lowpass filter. For this purpose, the filter was tuned with respect to previous studies addressing human motion characteristics [39] and human motion filtering [3, 40]. The cut-off frequency has been set to $1.5 \mathrm{~Hz}$ to preserve only fundamental hand motion frequencies, with a unity gain in the bandwidth and a high attenuation beyond $10 \mathrm{~Hz}[23]$. Practically, occlusion appearance was managed to stop the robot during the execution in order to ensure smooth and controlled motions. At the end, filtered signals are sent to the robot controller through an open UDP interface. Using the ROS middleware, motor control of the robotic arms is realized at $1 \mathrm{kHz}$ and both currents and torques are checked to fit in the required ranges for safe manipulation [1].

\section{Evaluation Study}

To compare both HMIs for robotic training, three operators were asked to execute a predefined surgical training task on the robotic platform presented in Section 3. By recording the surgical tool motions during the ex- 
ecution of the task, a dataset of trajectories is created for both interfaces (Section 4.1). From these trajectories, six metrics are computed to quantify performances for each interface (Section 4.2). Additionally, an unsupervised clustering approach is proposed to both analyse spatiotemporal differences between tool trajectories and provide a score describing not only global performance but local deviations also (Section 4.3).

\subsection{Trajectory Dataset}

A dataset of trajectories was acquired for both the Sigma. 7 interface and the Leap Motion. For each dataset, the same training task was executed five times by three different participants with different skill levels: an urologist expert who regularly performs surgeries with the da Vinci system (named ' $C$ '), a last year resident who only used few times a robotic training system (named ' $B$ ') and a teleoperation system engineer (named 'A'). All participants were right-handed. The objective behind integrating various experience into the experimental protocol is to analyse if the surgical experience affects the HMI comparison. Before experiments, participants were asked to sign consents regarding the study and were all briefed about the setup, its specificities and the task to ensure that they would perform it appropriately and consistently.

The surgical training task was directly inspired by the FLS guidelines [6]. This task involved peg transfers to several target locations following the workflow described hereafter:

1. Pick the first peg with the left tool and insert it into target 1 (leftmost pin of fig. 5),

2. Pick the second peg with the right tool and insert it into target 2 (rightmost pin),

3. Pick the last peg with the left or right tool and progress towards the center of the peg board. Grab it with the other available tool in order to insert it into target 3 (uppermost pin).

To ensure consistent acquisitions, 15 minutes of free training with each interface were allowed, and then each interface was selected randomly. Then, we acquired 10 trajectories for each participant with each HMI and computed the learning curve by taking into account the time to complete the task. Fitting a decreasing exponential model and reaching a plateau, we took the last five sessions for each configuration (i.e., participant and interface) where the overall average standard deviation (i.e., across all participants) regarding the time metric was 2.24 seconds for the Sigma.7 and reaches 3.68 seconds for the Leap Motion.

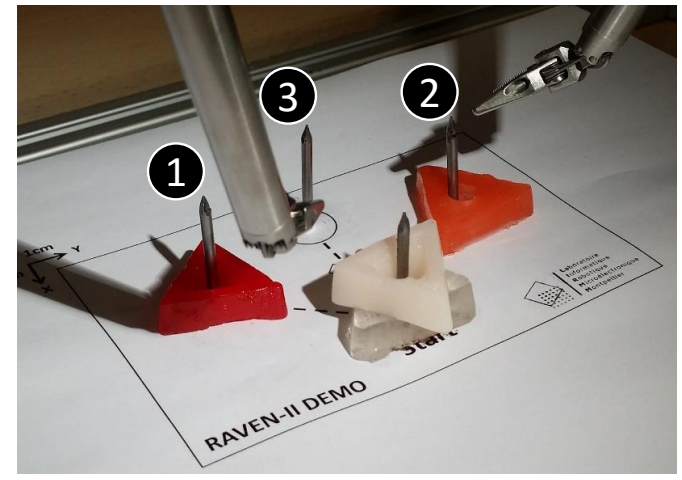

Fig. 5: The pick-and-place training task with three different target locations.

\subsection{Metric-based Analysis}

This metric-based comparison relies on the computation of the six following metrics that have been proposed to evaluate psychomotor skills in $[5,18,26]$. In the next, position of the robotic instrument is defined by $X=[x(t), y(t), z(t)]_{t=0}^{T}$ and velocity of the instrument by $v_{\text {left }}$ and $v_{\text {right }}$ for each hand.

1. Time $(T)$ corresponds to the execution time between the first time the foot pedal has been pressed to start the robot (i.e., clutch), until it has been released at the end of the task (i.e., unclutch). It is measured in seconds.

2. Bimanual Dexterity $(B D)$ measures the participant's ability to control both instruments at the same time. $\mathrm{BD}$ is found by computing the correlation between the velocity norm of the two instrument tool tips. It has to be noticed that bimanual dexterity is a subjective metric from the GOALS assessment method [37]. For an objective approach, the metric is computed from motion data [18].

$B D=\frac{\sum_{n=1}^{N}\left(v_{\text {left }}(n)-\bar{v}_{\text {left }}\right)\left(v_{\text {right }}(n)-\bar{v}_{\text {right }}\right)}{\sqrt{\sum_{n=1}^{N}\left(v_{\text {left }}(n)-\bar{v}_{\text {left }}\right)^{2} \sum_{n=1}^{N}\left(v_{\text {right }}(n)-\bar{v}_{\text {right }}\right)^{2}}}$

3. Path Length $(P L)$ is computed for both dominant and non-dominant hands and represents the total movement of the tool tip during the execution of the task. It is measured in meters:

$$
P L=\int_{0}^{T} \sqrt{\left(\frac{d x}{d t}\right)^{2}+\left(\frac{d y}{d t}\right)^{2}+\left(\frac{d z}{d t}\right)^{2} d t}
$$

4. Average Velocity $(A V)$ is computed for both dominant and non-dominant hands and corresponds to the average velocity norm measured at the tool tip of the instrument. It is measured in $\mathrm{m} / \mathrm{s}$. 
5. Motion Smoothness $(M S)$ is computed for both dominant and non-dominant hands and corresponds to the total jerk normalized by the duration of the task. MS is measured in $\mathrm{m} / \mathrm{s}^{3}$ :

$$
M S=\frac{J}{T}, J=\sqrt{\frac{1}{2} \int_{0}^{T}\left(\frac{d^{3} X}{d t}\right)^{2} d t}
$$

6. Working volume $(W V)$ is computed for both dominant and non-dominant hands and represents the volume of the convex hull for each trajectory. For this purpose, the Matlab function convhull() has been used. WV is measured in $\mathrm{m}^{3}$ :

The statistical analysis was realized using the MannWhitney $U$-test. A $p$-value $<0.05$ was considered as statistically significant (with $*$ for $p$-value $<0.05$, ** for $p$-value $<0.01$ and $* * *$ for $p$-value $<0.001)$.

\subsection{Spatiotemporal Analysis}

In this study, the trajectory shape is also analysed. Through this approach, the objective is to determine if the task execution from the robot point of view is similar from one interface to the other. Adapted from previous studies $[11,12]$, the following approach allows to cluster trajectories realized with both interfaces by taking into account their spatiotemporal similarities. Starting with the pool of trajectories defined by $X=$ $[x(t), y(t), z(t)]_{t=0}^{T}$ for each hand, the objective is to compute a global distance matrix. For this purpose, the Dynamic Time Warping (DTW) measure has been used for the spatiotemporal deformation measurement [29]. While the Euclidean distance cannot capture flexible similarities (see fig. 6), DTW allows to measure similarities between two sequences which may vary in time or speed. Its main advantage is the computation of a point-to-point association between two temporal sequences, with respect to both time and space variations. Thus, DTW finds the optimal alignment (or coupling) between sequences by aligning similar coordinates of both sequences. The cost of the optimal alignment between sequences $A=\left\langle a_{1}, \ldots, a_{M}\right\rangle$ and $B=\left\langle b_{1}, \ldots, b_{N}\right\rangle$ is recursively computed by:

$D\left(A_{i}, B_{j}\right)=\delta\left(a_{i}, b_{j}\right)+\min \left\{\begin{array}{l}D\left(A_{i-1}, B_{j-1}\right) \\ D\left(A_{i}, B_{j-1}\right) \\ D\left(A_{i-1}, B_{j}\right)\end{array}\right\}$

where $\delta\left(a_{i}, b_{j}\right)$ is the norm of the Euclidean distance between $a_{i}$ and $b_{j}$. The overall similarity of the two time series is given by $D\left(A_{|A|}, B_{|B|}\right)=D\left(A_{M}, B_{N}\right)$.

Practically, a multi-dimensional approach was employed to outperform the standard multiple one-dimensional

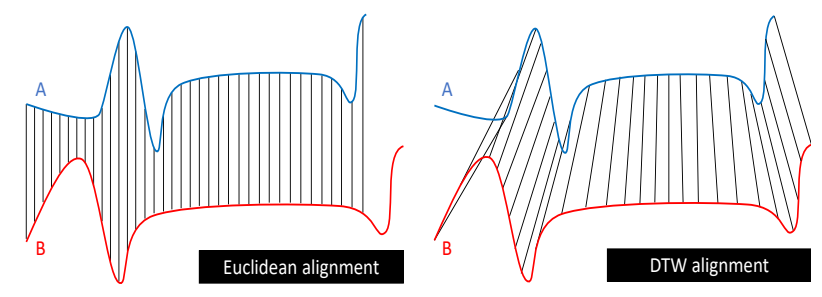

Fig. 6: Comparison between Euclidean and DTW alignments for two different time series.

alignment [32]. That is to say, the distance between two points (i.e., 3D points) corresponds to the euclidean norm and not only the sum of the distance of each point. Using such measure to compare two trajectories together, we constructed two different matrices (i.e., one for the left and one for the right hand) that contain the warped distance between each trial, from each participant. A third matrix was computed by adding the left and right distance matrices, which represents the sum of the warped distance of each hand for each trial.

The second step of the analysis consists in automatically cluster similar trajectories based on a global distance matrix. We used the unsupervised Hierarchical Agglomerative Clustering (HAC) [12] and computed the distance between clusters with the Ward method, which takes into account the inner squared distance (i.e., minimum variance algorithm).

\section{Experimental Results}

This section reports the results obtained for both metricbased and unsupervised clustering analyses. Data were acquired at the end of each training. Computation of all metrics and unsupervised trajectory analysis were run on Matlab R2016a with an Intel Xeon E5-1650V4 $@ 3.60 \mathrm{GHz}$.

\subsection{Metric-based Results}

From the three participants and the two HMIs handled for the comparison, a total of 30 acquisitions were performed (i.e., 5 per participant for each interface). Fig. 7 shows bar charts of the results from the Sigma.7 and the Leap Motion interfaces, for each experience group. Separated values for the instrument controlled by the dominant and the non-dominant hand are presented, whereas time and bimanual dexterity metrics refer for both hands.

Regarding the time metric (fig. 7a), a gap between the interfaces is visible with $p$-values of $0.00762,0.00878$ 
and 0.00207 respectively, whatever the experience is. Time to accomplish the task with the Leap Motion is significantly longer than using the mechanical Sigma.7 device, especially for the expert surgeon (C).

With the bimanual dexterity (fig. $7 \mathrm{~b}$ ), one can notice that except for the engineer(A) where the mean value is higher and the standard deviation larger with the Leap Motion, no significant difference appears. Only a small trend can be noticed for the resident (B) and expert surgeon (C) with a higher dexterity using the Sigma.7. Concerning the path length realized with the robotic instrument during the execution of the surgical training task, only significant results were obtained for the expert (C) with the non-dominant hand (fig. 7c). Here, the path followed by the instrument when using the Leap Motion was significantly higher than using the Sigma.7 ( $p$-value of 0.00817 ). For the dominant hand (fig. 7d), only larger standard deviations are shown for the resident (B) and the expert surgeon (C) but overall, there is no tendency in favor of one of the two interfaces. Taking into account the average velocity of the tip of the instruments when performing the pick-and-place task, the statistical test shows small $p$-values for both non-dominant hand (fig. 7e) with $0.00576,0.00843$ and 0.00612 respectively and dominant hand (fig. $7 \mathrm{f}$ ) with $0.00521,0.00988$ and 0.00175 . Lower standard deviations between sessions exist with the Leap Motion but the Sigma.7 device allows participants (especially expert surgeon $(\mathrm{C})$ ) to fully use their ability with both hands by changing the speed of their executions. At the end, the average velocity during the training task was reduced, which explains the increased time scores. However, even if significant differences have been shown for the average velocities during the task execution, computation of the motion smoothness shows no differences except for the expert surgeon (C) with both non-dominant (fig. 7g) and dominant hand (fig. 7h), where $p$-values reach 0.00878 and 0.00591 respectively. Globally, for both engineer (A) and resident (B), the smoothness is similar with both hands where for the expert, the Sigma.7 allows better execution and less bumps.

The last metric allows to compare the convex working volume from the surgical tools when using both the Sigma.7 and the Leap Motion. For the non-dominant hand (fig. 7i), only statistical difference is visible with the expert surgeon $(\mathrm{C})$ reaching a $p$-value of 0.00881 . Moreover, larger standard deviations can be observed with the non-dominant hands when using the Leap Motion. However, even if the difference with the dominant hand (fig. $7 \mathrm{j}$ ) is significant for both the engineer $(\mathrm{A})$ and the resident (B) ( $p$-values of 0.0244 and 0.0058), smaller deviations are visible especially for the expert surgeon (C) with no statistical difference.

From this first analysis, one can state that regarding both execution time and average velocity metrics, the Leap Motion does not offer the same regularity than the Sigma.7 during the execution of standard gestures. Conversely, bimanual dexterity and path length do not highlight any differences in terms of execution when performing with both interfaces. Where 7 of the 10 scores obtained from the expert surgeon express a clear tendency in favor of the mechanical Sigma.7, engineer and especially resident scores do not show such trend. Overall, the performance with the Leap Motion is not similar when the surgical experience changes and close performance between both interface are observed when participant does not have any prior experience.

\subsection{Spatiotemporal Results}

This analysis has been done on the 30 acquisitions from the three candidates named 'A', 'B' and 'C'. Each candidate performed five sessions with each interface, numbered from 1 to 5 . Also the interface used during manipulation is referred as a letter: 'S' for the Sigma. 7 and 'L' for the Leap Motion. Fig. 8a shows the dendrogram from the proposed method which combines the DTW measure and the HAC clustering. Six low-level clusters appear when coloring each similarity group.

Regarding the aptitude of each candidate, the urologist surgeon ('C') performed differently with both Sigma.7 and Leap Motion interfaces (i.e., blue and red clusters respectively). However, the warping distance (i.e., distance between colored clusters) allows to conclude that the intra-observer variability is low and then the HMI does not highly impact his motions. It means that a similar spatiotemporal strategy was used for both the Sigma.7 and the Leap Motion interfaces. Moreover, one can note that clusters from candidate ' $\mathrm{C}$ ' with the Leap Motion (i.e., red cluster) and from candidate 'A' with the Leap Motion (i.e., yellow cluster) are directly linked meaning that they adopted similar strategies also, which are however separable as it can be visually expressed in fig. 8b. With the same candidates, the distinction with the Sigma.7 contact-based control is stronger and different strategies were adopted as it is shown in fig. 8c. Conversely, clusters from candidates ' $\mathrm{A}$ ' and ' $\mathrm{B}$ ' with both the Sigma.7 interface are linked (i.e., purple and cyan clusters respectively), indicating a strong relation in their execution. However, candidate 'B' with the Leap Motion (i.e., green cluster) performed differently from the other configurations (i.e., separated green cluster). When looking at the warping distances, we can notice that using the contactless control seems to impact differently the spatiotemporal strategy, depending 


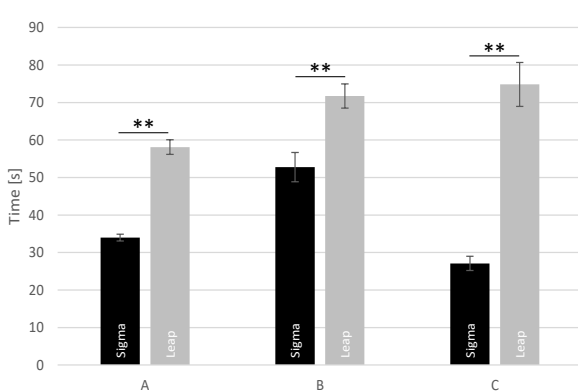

(a) Execution time.

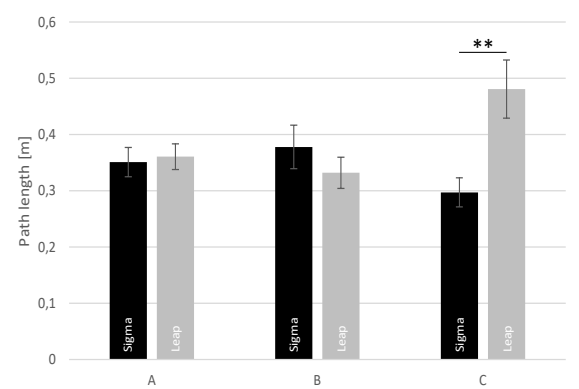

(c) Non-dominant hand path length.

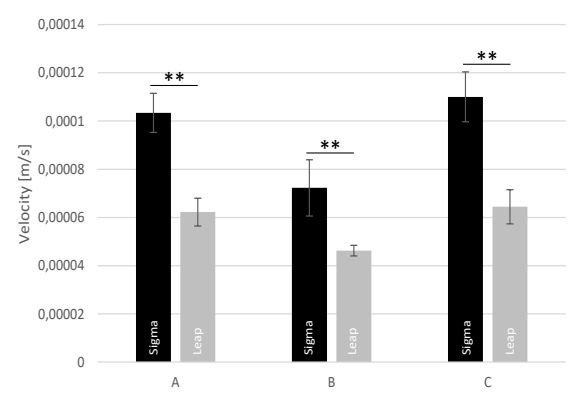

(e) Non-dominant hand average velocity.

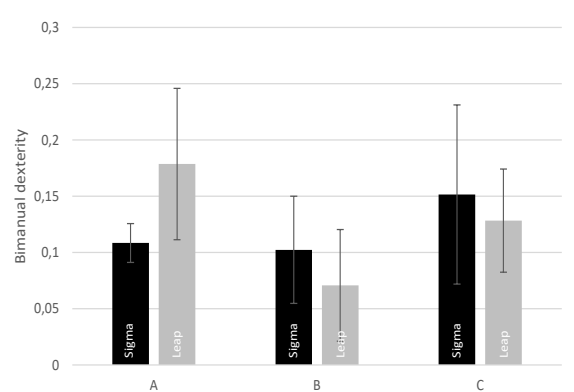

(b) Bimanual dexterity.

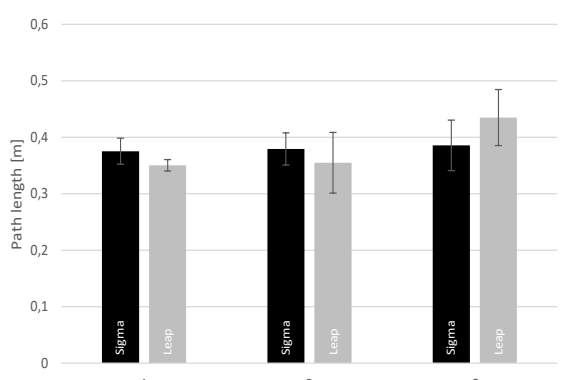

(d) Dominant hand path length.

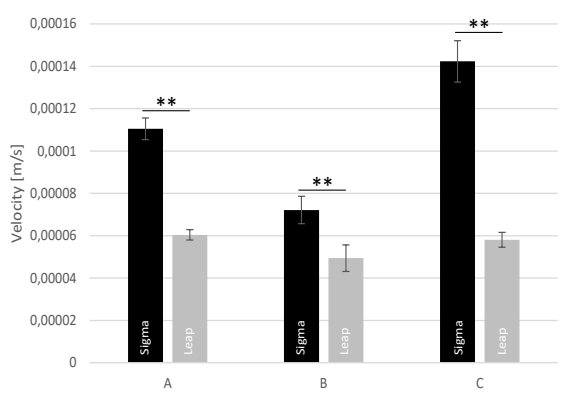

(f) Dominant hand average velocity.

Fig. 7: (a)-(j) Bar charts representation of the computed metrics from the instrument trajectories when using both the Sigma.7 (in black) and the Leap Motion (in grey), for each experience group (A = Engineer, B = Resident, C $=$ Expert surgeon).

on the experience (especially for the resident). Looking at the clusters, it can be noticed that each candidate is distinctly separated from the other ones. In addition, each cluster highlights one of the two HMI, indicating that trajectories are similar for the same configuration (i.e., participant and interface), but different enough between configurations to be distinguished.

\section{Discussions}

The discussion is splitted in two parts. The first part directly focuses on the results interpretations whereas the second draws up perspectives of the presented work and future improvements.

\subsection{Results Interpretation}

The metric-based analysis highlights significant differences especially for the surgeon that can be mainly explained by three factors. The first one is that contactless interaction is difficult to manage especially when performing a grasping task. Where the Sigma.7 offers tactile perception and supports arm proprioception thanks to the mechanical handle, free-hand interaction is more complex and requires more time to practice. This type of interaction led trainee to use the clutch in order to pause the session for looking their hands and place them correctly. The second factor addresses workspace limitations. It appeared several times during the experiments that participants stopped unintentionally the robot due to a lack of the workspace perception. Their 


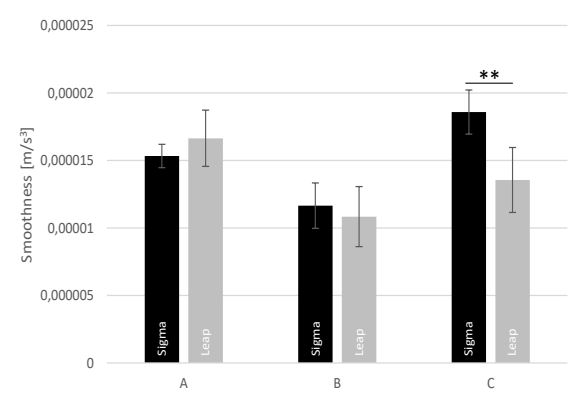

(g) Non-dominant hand smoothness.

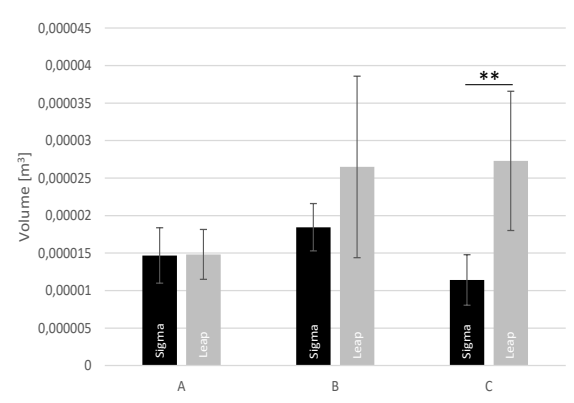

(i) Non-dominant hand working volume.

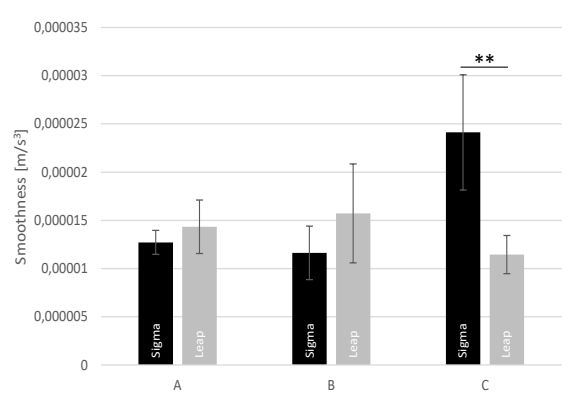

(h) Dominant hand smoothness.

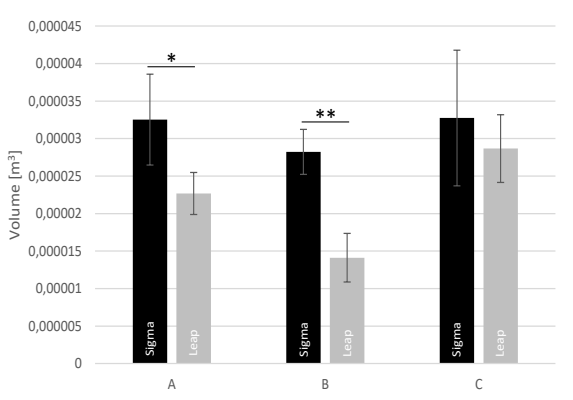

(j) Dominant hand working volume.

Fig. 7: Continued.

hands cross the tracking limits that caused robot interruption. The last factor deals with hand occlusions. For safe control, the current implementation manages hand occlusions to instantaneously stop the robot in order to avoid unsafe motions and arm collision. In the three cases (i.e., proprioception, workspace limits and occlusions), after an interruption, the robot was able to start again at the same place and allowed a smooth manipulation recovery. However, such event appearances increased the execution time and reduced average velocities obtained with the contactless control.

From the spatiotemporal analysis, we notice that taking motion of the surgical tooltips into account enables detection of both participant and interface in an unsupervised way. Consequently, it seems that the current configuration (i.e., participant and interface) produces a specific signature in the trajectory that allows detection of each of them. From the hardware point of view, this result suggests that participants did not follow the same strategy when using each interface, meaning that manipulation and interactions with their hands could be different for both devices.

However, thank to these two complementary analyses, one can state that this specific trajectory signature is not comparable for the surgeon and for the resident. Where the experienced participant produced lower warping distances but highlighted significant differences in 7 of the 10 computed metrics, the novice can be distinguished by lower variations in the metric analysis and larger ones in the spatiotemporal clustering. This result supports the hypothesis that similar manipulability is not mandatory for the design of HMIs dedicated to surgical training and suggests that complementary to metric-based computations, other analysis should be perform to qualify and quantify the surgical performance during robotic-assisted training.

\subsection{Perspectives}

This work shows promising potentials for new telesurgical control systems. By using the Leap Motion device to accurately control surgical instruments, we opened new perspectives for future developments of contactless-based interface dedicated to robotic surgical training systems. While stable control for safety protection is mandatory in the OR, current occlusion handling strategy which consists in stopping the robot led participants to clutch more and then reduce their scores. Providing a more detailed analysis of the different gesture workflows performed during the surgical task could help to better understand variations between contactless and contact-based control methods. For this purpose, approaches could rely on Surgical Process Models that allow to breakdown a process into different granularity levels that are steps, phases and activities [11, 12, 22]. In the current implementation, manipulation capabilities are mainly restricted because of the occlusion strat- 


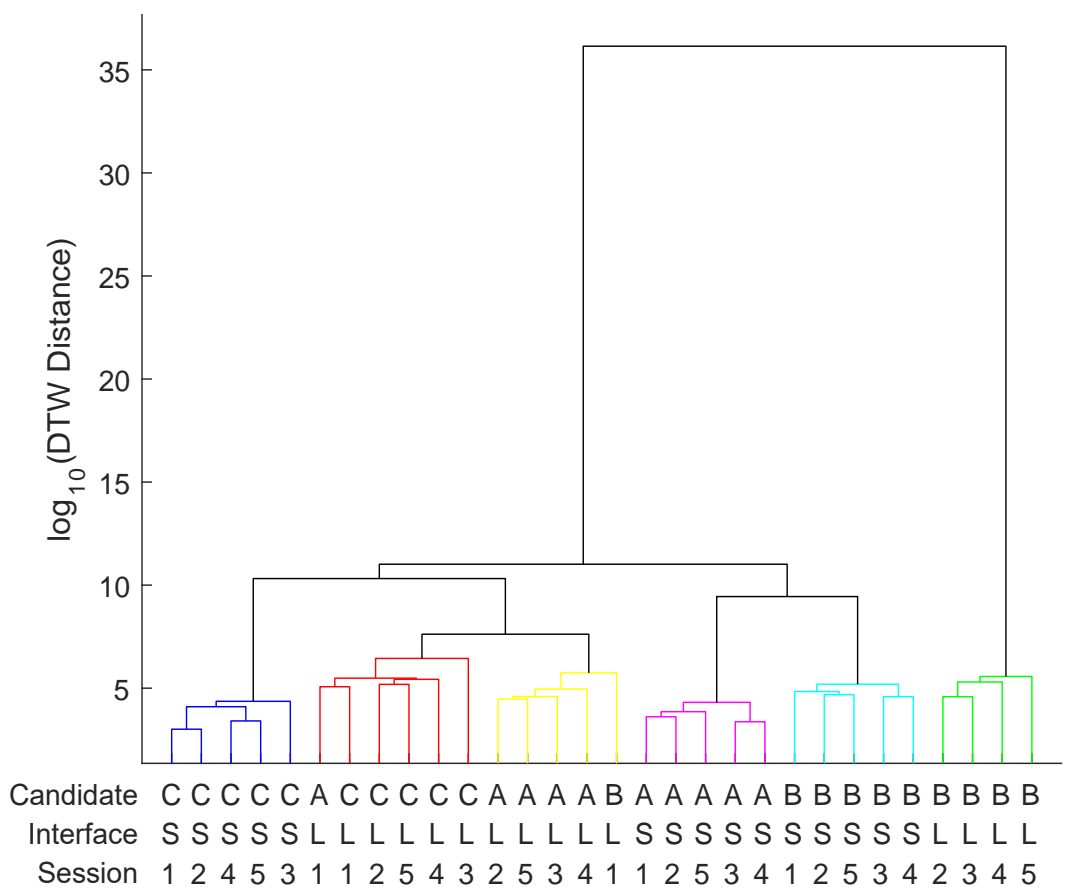

(a) Dendrogram showing the trajectory similarities between candidates and interfaces with DTW + HAC. Each candidate is referred to a letter ('A', 'B' and 'C') as well as the Sigma.7 interface ('S') and the Leap Motion ('L').

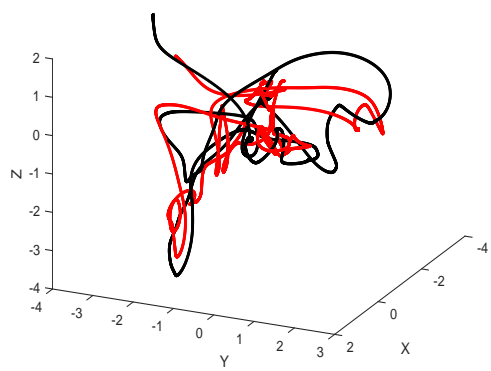

(b) Low-variability trajectories from engineer (black) and expert (red) using the Leap Motion.

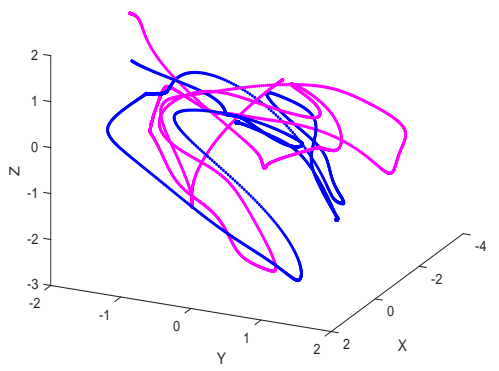

(c) Low-variability trajectories from engineer (magenta) and expert (blue) with the Sigma.7.

Fig. 8: Unsupervised trajectory analysis results with (a) the clustering of each session from each candidate using both interfaces and (b) and (c) show examples of respectively similar and dissimilar surgical tool trajectories from dominant hands, acquired during training sessions.

egy, the workspace limitations and the proprioception perceptions. To tackle these issues, complementary informations should be provided to the operator such as visual, auditive or tactile feedbacks [19]. Despite these drawbacks, the Leap Motion device offers several advantages in comparison to mechanical-based interfaces. It is drastically cheaper than two Sigma.7 (i.e., for bimanual teleoperation), which helps improving availability of training systems for continuous teaching of surgical skills. Also, its portability and ease of use make it easily adaptable to different models of training systems (i.e., virtual reality simulator or robot). Considering perioperative situations, the contactless control offers sterilization compatibility that simplifies the workflow in case of medical complications. However, even if it does not provide similar bimanual workspace and raw accuracy, this contactless approach is accurate enough to control surgical simulators and robots, for training purposes only when complex motions are not always required $[21,38]$.

To support our conclusion that the Leap Motion could be a reliable alternative to high-end quality interface for training purposes, additional surgical tasks requiring higher accuracy such as dissection of small anatomical structures, suturing and clip application could be studied to extend the current results. Experiments including more participants with different skill levels could help to generalize our conclusion. The natural next steps of this work is first to integrate such contactless control into a training system and compare the performance with its original human-machine interface in order to obtain a reliable training tool. According to the preliminary results, the second step will be a transfer study towards clinical practice, where surgeons will be trained with the contactless system and then tested for improvement 
in standard teleoperation, compared to no training and other forms of training.

\section{Conclusion}

Surgical simulation is a key component in the initial education of surgeons, especially for robotic-assisted interventions. In such context, more and more advanced systems are developed with high-end mechanical humanmachine interfaces. In this paper, we proposed to replace such expansive interface by using a low-cost device, namely the Leap Motion, to perform telesurgical training with a Raven-II robot. Relying on an efficient hand model from the Leap Motion tracker, we can fully and accurately manipulate surgical instruments. Here, one hand mimics one surgical tool with 7 degrees of freedom, including grasp action. Then, we assessed the proposed approach by comparing performance of several participants on a peg transfer task. From a metric-based quantification, results showed that the Leap Motion based control performed differently than the mechanical control reference. In addition, a novel unsupervised clustering approach was used to compare spatiotemporal trajectories of the surgical instruments with both devices. At the end, we concluded that strategies and motions are distinct and the contactless device does not overtake manipulability with high-end mechanical interface. However, current development highlights that accurate control of robotic surgical tools is feasible and could be dedicated for training purposes. Considering cost, dimension, accuracy and asepsis, the contactless control should be emphasized for novel generation of robotic surgical training system to help trainee acquiring basic skills and encourage the educational heuristic "perfect practice makes perfect".

\section{Compliance with Ethical Standards}

Conflict of interest The authors declare that they have no conflict of interest.

Ethical approval All procedures performed in studies involving human participants were in accordance with the ethical standards of the institutional and/or national research committee and with the 1964 Helsinki Declaration and its later amendments or comparable ethical standards.

Informed consent Informed consent was obtained from all individual participants included in the study.

\section{References}

1. Alemzadeh H, Chen D, Lewis A, Kalbarczyk Z, Raman J, Leveson N, Iyer R (2015) Systems-theoretic safety assessment of robotic telesurgical systems. International
Conference on Computer Safety, Reliability, and Security 9337:213-227

2. Alemzadeh H, Raman J, Leveson N, Kalbarczyk Z, Iyer RK (2016) Adverse events in robotic surgery: A retrospective study of 14 years of FDA data. PLOS ONE $11(4): 1-20$

3. Casiez G, Roussel N, Vogel D (2012) 1 Filter: A Simple Speed-based Low-pass Filter for Noisy Input in Interactive Systems. ACM Annual Conference on Human Factors in Computing Systems pp 2527-2530

4. Cooper MA, Ibrahim A, Lyu H, Makary MA (2015) Underreporting of Robotic Surgery Complications. Journal for Healthcare Quality 37(2):133-138

5. Cotin S, Stylopoulos N, Ottensmeyer MP, Neumann PF, Rattner DW, Dawson S (2002) Metrics for Laparoscopic Skills Trainers: The Weakest Link! Medical Image Computing and Computer-Assisted Intervention 2488:35-43

6. Derossis AM, Fried GM, Abrahamowicz M, Sigman HH, Barkun JS, Meakins JL (1998) Development of a Model for Training and Evaluation of Laparoscopic Skills. The American Journal of Surgery 175(6):482-487

7. Despinoy F, Alonso S, Zemiti N, Jannin P, Poignet P (2014) Comparative assessment of a novel optical human-machine interface for laparoscopic telesurgery. International Conference on Information Processing in Computer-Assisted Interventions 8498:21-30

8. Dragan AD, Srinivasa SS, Lee KCT (2013) Teleoperation with Intelligent and Customizable Interfaces. Journal of Human-Robot Interaction 2(2):33-57

9. Du G, Zhang P, Mai J, Li Z (2012) Markerless KinectBased Hand Tracking for Robot Teleoperation. International Journal of Advanced Robotic Systems 9(36):1-10

10. Forestier G, Lalys F, Riffaud L, Trelhu B, Jannin P (2011) Assessment of surgical skills using Surgical Processes and Dynamic Time Warping. Modeling and Monitoring of Computer Assisted Interventions Workshop pp $1-10$

11. Forestier G, Lalys F, Riffaud L, Trelhu B, Jannin P (2012) Classification of surgical processes using dynamic time warping. Journal of Biomedical Informatics $45(2): 255-264$

12. Forestier G, Lalys F, Riffaud L, Louis Collins D, Meixensberger J, Wassef SN, Neumuth T, Goulet B, Jannin P (2013) Multi-site study of surgical practice in neurosurgery based on surgical process models. Journal of Biomedical Informatics 46(5):822-829

13. Freschi C, Ferrari V, Melfi F, Ferrari M, Mosca F, Cuschieri A (2013) Technical review of the da Vinci surgical telemanipulator. The International Journal of Medical Robotics and Computer Assisted Surgery 9(4):396-406

14. Guna J, Jakus G, Pogačnik M, Tomažič S, Sodnik J (2014) An Analysis of the Precision and Reliability of the Leap Motion Sensor and Its Suitability for Static and Dynamic Tracking. Sensors 14(2):3702-3720

15. Guthart G (2016) Intuitive Surgical 2016 Annual Report

16. Hannaford B, Rosen J, Friedman DW, King H, Roan P, Cheng L, Glozman D, Ma J, Kosari SN, White L (2013) Raven-II: An Open Platform for Surgical Robotics Research. IEEE Transactions on Biomedical Engineering 60(4):954-959

17. Hernoux F, Béarée R, Gajny L, Nyiri E, Bancalin J, Gibaru O (2013) Leap Motion pour la capture de mouvement 3D par spline L1 - Application à la robotique. Conférence Groupe de Travail en Modélisation Géométrique pp 1-6 
18. Hofstad EF, Våpenstad C, Chmarra MK, Lang $\varnothing \mathrm{T}$, Kuhry E, Mårvik R (2013) A study of psychomotor skills in minimally invasive surgery: What differentiates expert and nonexpert performance. Surgical Endoscopy $27(3): 854-863$

19. Howard T, Szewczyk J (2016) Improving Precision in Navigating Laparoscopic Surgery Instruments toward a Planar Target Using Haptic and Visual Feedback. Frontiers in Robotics and AI 3:37

20. Jin H, Chen Q, Chen Z, Hu Y, Zhang J (2016) MultiLeapMotion sensor based demonstration for robotic refine tabletop object manipulation task. Transactions on Intelligence Technology 1(1):104-113

21. Kim Y, Kim PCW, Selle R, Shademan A, Krieger A (2014) Experimental Evaluation of Contact-Less Hand Tracking Systems for Tele-Operation of Surgical Tasks. IEEE International Conference on Robotics and Automation pp 3502-3509

22. Lalys F, Jannin P (2014) Surgical process modelling: A review. International Journal of Computer Assisted Radiology and Surgery 9(3):495-511

23. Loram ID, Gawthrop PJ, Lakie M (2006) The frequency of human, manual adjustments in balancing an inverted pendulum is constrained by intrinsic physiological factors. The Journal of physiology 577(1):417-432

24. Masson-Lecomte A, Bensalah K, Seringe E, Vaessen C, de la Taille A, Doumerc N, Rischmann P, Bruyre F, Soustelle L, Droupy S, Rouprt M (2013) A prospective comparison of surgical and pathological outcomes obtained after robot-assisted or pure laparoscopic partial nephrectomy in moderate to complex renal tumours: results from a French multicentre collaborative study. British Journal of Urology 111(2):256-263

25. Moglia A, Ferrari V, Morelli L, Ferrari M, Mosca F, Cuschieri A (2016) A Systematic Review of Virtual Reality Simulators for Robot-assisted Surgery. European Urology 69(6):1065-1080

26. Oropesa I, Sánchez-González P, Lamata P, Chmarra MK, Pagador JB, Sánchez-Margallo Ja, Sánchez-Margallo FM, Gómez EJ (2011) Methods and Tools for Objective Assessment of Psychomotor Skills in Laparoscopic Surgery. Journal of Surgical Research 171(1):81-95

27. Rossol N, Cheng I, Basu A (2016) A Multisensor Technique for Gesture Recognition Through Intelligent Skeletal Pose Analysis. IEEE Transactions on HumanMachine Systems 46(3):350-359

28. Sachdeva AK, Buyske J, Dunnington GL, Sanfey HA, Mellinger JD, Scott DJ, Satava R, Fried GM, Jacobs LM, Burns KJ (2011) A New Paradigm for Surgical Procedural Training. Current problems in surgery 48(12):854-968

29. Sakoe H, Chiba S (1978) Dynamic Programming Algorithm Optimization for Spoken Word Recognition. IEEE Transactions on Acoustics, Speech, and Signal Processing 26(1):43-49

30. Schreuder HWR, Wolswijk R, Zweemer RP, Schijven MP, Verheijen RHM (2012) Training and learning robotic surgery, time for a more structured approach: A systematic review. BJOG: An International Journal of Obstetrics and Gynaecology 119(2):137-149

31. Simorov A, Otte RS, Kopietz CM, Oleynikov D (2012) Review of surgical robotics user interface: what is the best way to control robotic surgery? Surgical Endoscopy 26(8):2117-2125

32. Ten Holt GA, Reinders MJT, Hendriks EA (2007) MultiDimensional Dynamic Time Warping for Gesture Recognition. Annual Conference of the Advanced School for
Computing and Imaging 5:1-8

33. Tobergte A, Helmer P, Hagn U, Rouiller P, Thielmann S, Grange S, Albu-Schäffer A, Conti F, Hirzinger G (2011) The sigma.7 haptic interface for MiroSurge: A new bimanual surgical console. IEEE International Conference on Intelligent Robots and Systems pp 3023-3030

34. Travaglini TA, Swaney PJ, Weaver KD, Webster III RJ (2015) Initial Experiments with the Leap Motion as a User Interface in Robotic Endonasal Surgery. International Symposium on Robotics and Mechatronics 37:171179

35. Tsuda S, Scott D, Doyle J, Jones DB (2009) Surgical Skills Training and Simulation. Current problems in surgery $46(4): 271-370$

36. Vargas HF, Vivas OA (2014) Gesture recognition system for surgical robot's manipulation. Symposium on Image, Signal Processing and Artificial Vision pp 1-5

37. Vassiliou MC, Feldman LS, Andrew CG, Bergman S, Leffondré K, Stanbridge D, Fried GM (2005) A global assessment tool for evaluation of intraoperative laparoscopic skills. American Journal of Surgery 190(1):107-113

38. Weichert F, Bachmann D, Rudak B, Fisseler D (2013) Analysis of the accuracy and robustness of the Leap Motion Controller. Sensors 13(5):6380-6393

39. Xiong Y, Quek F (2006) Hand Motion Gesture Frequency Properties and Multimodal Discourse Analysis. International Journal of Computer Vision 69(3):353-371

40. Yang S, Wells TS, Maclachlan RA, Riviere CN (2013) Performance of a 6-Degree-of-Freedom Active Microsurgical Manipulator in Handheld Tasks. IEEE International Conference on Engineering in Medicine and Biology Society 2013:5670-5673

41. Zhou T, Cabrera ME, Wachs JP (2014) Touchless telerobotic surgery: A comparative study. Workshop on Telerobotics for Real-Life Applications, Challenges and New Developments, in Intelligent Robots and Systems

42. Zhou T, Cabrera ME, Low T, Sundaram C, Wachs J (2016) A Comparative Study for Telerobotic Surgery Using Free Hand Gestures. Journal of Human-Robot Interaction 5(2):1-28 